Management international

International Management

Gestiòn Internacional

Entre idéal du projet et consensus idéal, différentes approches de la gouvernance démocratique des organisations à but non-lucratif

Between the Ideal of the Project and Ideal Consensus: Different Approaches to Democratic Governance in Nonprofit Organizations Entre el ideal del proyecto y el consenso ideal, diferentes concepciones de la gobernanza democrática de las organizaciones sin fines de lucro

Patrick Valeau, Hassen Parak, Frédéric Annette et Jérôme Boncler

Volume 24, numéro 1, 2020

URI : https://id.erudit.org/iderudit/1069092ar

DOI : https://doi.org/10.7202/1069092ar

Aller au sommaire du numéro

Éditeur(s)

HEC Montréal

Université Paris Dauphine

ISSN

1206-1697 (imprimé)

1918-9222 (numérique)

Découvrir la revue

Citer cet article

Valeau, P., Parak, H., Annette, F. \& Boncler, J. (2020). Entre idéal du projet et consensus idéal, différentes approches de la gouvernance démocratique des organisations à but non-lucratif. Management international / International Management / Gestiòn Internacional, 24(1), 30-42.

https://doi.org/10.7202/1069092ar
Résumé de l'article

La gouvernance des organisations à but non lucratif est fondée sur le principe démocratique " un homme, une voix ». Nos trente entretiens restituent un continuum allant de l'« idéal du projet » au " consensus idéal ». L’idéal du projet rejoint la fonction disciplinaire de la gouvernance dans le cadre de laquelle les présidents défendent la réalisation efficiente du projet validée par la majorité. Le consensus idéal correspond à la fonction cognitive suivant laquelle les présidents laissent le projet évoluer en fonction des différents points de vue exprimés au sein de l'assemblée afin d'obtenir l'adhésion de tous.
Tous droits réservés (C) Management international / International Management / Gestión Internacional, 2020
Ce document est protégé par la loi sur le droit d'auteur. L’utilisation des services d’Érudit (y compris la reproduction) est assujettie à sa politique d'utilisation que vous pouvez consulter en ligne.

https://apropos.erudit.org/fr/usagers/politique-dutilisation/ 


\section{Entre idéal du projet et consensus idéal, différentes approches de la gouvernance démocratique des organisations à but non-lucratif}

\section{Between the Ideal of the Project and Ideal Consensus: Different Approaches to Democratic Governance in Nonprofit Organizations}

\section{Entre el ideal del proyecto y el consenso ideal, diferentes concepciones de la gobernanza democrática de las organizaciones sin fines de lucro}
PATRICK VALEAU
IAE de la Réunion

\author{
HASSEN PARAK
IAE de la Réunion \\ HASSEN PARAK
IAE de la Réunion
}
FRÉDÉRIC ANNETTE
IAE de la Réunion

\section{JÉRÔME BONCLER \\ Université de Bordeaux-IUT \\ Département Technique de \\ Commercialisation}

\begin{abstract}
RÉSUMÉ
La gouvernance des organisations à but non lucratif est fondée sur le principe démocratique "un homme, une voix». Nos trente entretiens restituent un continuum allant de l' «idéal du projet» au "consensus idéal». L'idéal du projet rejoint la fonction disciplinaire de la gouvernance dans le cadre de laquelle les présidents défendent la réalisation efficiente du projet validée par la majorité. Le consensus idéal correspond à la fonction cognitive suivant laquelle les présidents laissent le projet évoluer en fonction des différents points de vue exprimés au sein de l'assemblée afin d'obtenir l'adhésion de tous.
\end{abstract}

Mots-Clés : OBNL, président, gouvernance, démocratie, conflits

\section{ABSTRACT}

The governance of non-profit organizations is based on the democratic principle of "one man, one voice". Our thirty interviews revealed a range from the "ideal of the project" to the "ideal consensus". The ideal of the project connects the disciplinary function of the governance in which the presidents defend the efficient realization of the project supported by the majority. The ideal consensus corresponds to a cognitive approach to governance according to which the presidents allow the project to evolve according to the different points of view expressed within the assembly in order to obtain the commitment of all.

Keywords: Nonprofit organizations, president, governance, democracy, conflicts
RESUMEN

La gobernanza de las organizaciones sin fines de lucro se basa en el principio democrático de "un hombre, una voz". Nuestras treinta entrevistas restauran un continuo que va desde el "ideal del proyecto" hasta el "consenso ideal". El ideal del proyecto se une a la función disciplinaria de gobierno en la cual los presidentes defienden la realización eficiente del proyecto validado por la mayoría. El consenso ideal corresponde a la función cognitiva según la cual los presidentes dejan que el proyecto evolucione según los diferentes puntos de vista expresados dentro de la asamblea para obtener la adhesión de todos.

Palabras Clave: Organizaciones sin fines de lucro, presidente, gobierno, democrático, conflictos a "gouvernance démocratique» constitue l'un des trois
critères retenus par les principaux groupes de recherche
internationaux (Hopkins; ISTR; EMES; ARNOVA) pour définir
les organisations à but non lucratif. Ces groupes considèrent
ainsi des organisations (1) productrices de biens et services; (2)
avec une finalité sociale visant à prendre en charge des publics
exclus ou des problèmes ignorés et (3) caractérisées par une
gouvernance démocratique garantissant leur indépendance
vis-à-vis des pouvoirs publics et des marchés. Cette gouver-
nance démocratique est souvent instituée dans le cadre de
lois générales indiquant que le conseil d'administration et le
président sont élus par une assemblée générale, composée des
parties prenantes membres de l'OBNL, suivant le principe «un
homme, une voix». Ces "mécanismes intentionnels internes"
de la gouvernance (Charreaux, 2003) doivent normalement
aboutir à l'établissement d'un projet ayant une fonction à
la fois cognitive et disciplinaire. Le projet peut être défini comme l'actualisation de la mission sous la forme d'objectifs opérationnels à court et moyen termes associés à des moyens plus précisément identifiés (Hoarau et Laville, 2008; Biondi, Chatelain, Eynaud et Sponem, 2010; Boncler et Valéau, 2010). Le projet s'apparente ainsi à une première forme de planification stratégique guidant les actions du président et du conseil d'administration, mais aussi les initiatives et les innovations sociales de l'ensemble des participants, y compris notamment celles du directeur, des salariés et des bénévoles (Valéau, 1999; Valéau, Eynaud, Chatelain-Ponroy et Sponem, 2018).

La littérature sur les OBNL introduit un cadre d'analyse utile, mais ne fournit que peu de connaissances concernant la façon dont cette gouvernance démocratique est mise à l'épreuve de l'action. Les études de cas portant sur d'autres thématiques, qu'elles soient anglo-saxonnes (Kelley, Lune \& Murphy, 2005; Kreutzer \& Jager, 2011) ou francophones (Hoarau \& Laville, 
2008; Boncler \& Valéau, 2010; Valéau \& Annette, 2012) montrent souvent des tensions susceptibles d'émerger entre différents groupes d'acteurs internes, y compris parfois au sein même du conseil d'administration (Valéau, Parak \& Louart, 2013). Ces différents acteurs sont tout aussi impliqués, mais ne partagent simplement pas la même vision du développement de leur OBNL (Drucker, 1990, Laville \& Glémain, 2009; Boncler \& Valéau, 2010). Ces différends sont «normaux» et constituent le corollaire logique des différentes orientations possibles des OBNL (Valéau, 2003, 2015) et de la diversité des acteurs impliqués dans la prise de décision démocratique (Herman \& Renz, 1997; Hoarau \& Laville, 2008; Valéau, 2003; 2015). Cependant, ces différends et les conflits qui peuvent en résulter mettent à mal la capacité de l'assemblée générale et du conseil d'administration à fournir un projet partagé par tous. Ce problème n'est pas ou peu étudié par la littérature sur la gouvernance des OBNL.

La question examinée dans le cadre de cette recherche ne concerne pas le fonctionnement ordinaire de la gouvernance des OBNL, mais porte sur la façon dont les présidents opèrent pour restaurer les mécanismes de la gouvernance des OBNL, lorsque ces derniers sont enrayés par les différends et les conflits entre les parties prenantes présentes au sein de l'assemblée générale et du conseil d'administration. En pratique, le principe « un homme une voix " peut être interprété tour à tour comme un droit de vote ponctuel ou comme un droit à la parole plus permanent. Nous portons ainsi notre attention sur les relations entre le président et les membres de l'assemblée générale. Plus précisément, nous nous focalisons sur les arbitrages souvent implicites effectués par le président, à l'intérieur des cadres définis par les membres de l'assemblée, afin de réguler ces différends. Nous montrons que, lors de son élection, puis entre deux renouvellements de son mandat, c'est souvent lui qui décide de prendre en compte ou non les voix discordantes au sein de l'assemblée générales dans la définition du projet mis en œuvre. L'apprentissage de la gouvernance des OBNL par les présidents consiste ainsi, en premier lieu, à accepter les désaccords et à faire le deuil du double idéal d'un collectif uni au service d'une même cause; il implique ensuite de comprendre qu'il n'y a pas d'autre choix que d'arbitrer en faveur de son projet ou du collectif.

Suivant une démarche empirico-formelle, sur la base de nos trente entretiens, nous montrons que ces situations obligent les présidents à se positionner le long d'un continuum situé entre deux approches :

- «l'idéal du projet» qui consiste pour le président à défendre la réalisation la plus efficiente possible de sa vision du projet, celle pour laquelle il a été élu. Cette approche s'appuie sur la «fonction disciplinaire» de l'assemblée générale;

- «l'idéal consensuel» dans le cadre duquel le président s'attache à débattre et redéfinir les différents aspects du projet avec l'ensemble des parties prenantes de façon que tous en reconnaissent la légitimité. Cette approche rejoint la «fonction cognitive» de l'assemblée.

Nous retrouvons ce faisant, au cœur de la gouvernance démocratique des OBNL, des interrogations relativement similaires à celles des sciences politiques concernant les pratiques de la démocratie au sein de nos sociétés modernes entre, d'un côté, une "démocratie représentative», efficiente mais qui tend à déresponsabiliser les citoyens et, de l'autre, des formes de «démocratie participative» introduisant un débat légitime mais techniquement difficile à maintenir (Blondiaux, 2008; Callon, Lascoumes \& Barthe, 2001; Koeble, 2006).

L'intérêt de cet article est de contribuer, à travers les cas d'un certain nombre d'OBNL françaises, à la réflexion, internationalement menée, sur les spécificités de la gouvernance dans les OBNL. On sait désormais que ces organisations représentent dans le monde $7 \%$ de l'emploi et $5 \%$ des PIB (Organisation des Nations Unies, Hopkins, 2003). Partant de là, la tentation est grande de les aborder comme des entreprises comme les autres et de dupliquer les normes issues du monde de l'entreprise. Eynaud (2015), Hoarau et Laville (2018), Boncler et Valéau (2015) mettent cependant en avant des spécificités telles que la notion de bien commun et une création de valeur multicritères nécessitant d'adapter les cadres établis pour les entreprises privées. Suivant une approche similaire à celle de Le Tian et Venard (2012), nous formalisons une diversité de modèles suivant lesquels les présidents établissent progressivement des formes socialement construites de la gouvernance démocratique dans les OBNL.

Nous commençons par une revue de la littérature appliquant les cadres d'analyse établis par Charreaux aux spécificités des OBNL puis en les confrontant à la notion de désaccords. Nous présentons ensuite notre méthode et nos résultats, puis discutons ces derniers avec une attention particulière pour les recommandations auprès de ces dirigeants.

\section{Cadre théorique}

\section{LES SPÉCIFICITÉS DE LA GOUVERNANCE DES OBNL}

Charreaux $(1997 ; 2003 ; 2006)$ définit la gouvernance comme un ensemble de mécanismes visant à réduire les biais décisionnels des dirigeants. Charreaux (2003) identifie deux types de biais : des biais disciplinaires, dit d'agence, suivant lequel le dirigeant pourrait adopter des comportements opportunistes et des biais cognitifs liés aux limites des compétences et des connaissances du dirigeant. L'approche disciplinaire de la gouvernance consiste ainsi à limiter les pouvoirs décisionnels des dirigeants et à cadrer leurs initiatives à travers des systèmes de récompenses mais aussi de sanctions, formels et informels, elle s'assure que les orientations suivies et les innovations introduites sont bien conformes aux intérêts de l'entreprise. L'approche cognitive de la gouvernance vise quant à elle le développement de nouvelles opportunités et la création de valeur à travers les apprentissages organisationnels et l'innovation (Charreaux, 2003. Elle a pour objectif «d'exploiter au mieux la connaissance spécifique répartie entre les différents acteurs, de veiller à son allocation optimale» (Charreaux, 2003, p. 11). Charreaux (2003) suggère ainsi de considérer l'approche partenariale et la dimension cognitive comme relevant d'un seul et même modèle visant à la fois la pacification des relations avec les différentes parties prenantes et la réduction des biais décisionnels. La gouvernance partenariale et cognitive se fonderait ainsi sur «la variété de ressources cognitives que chaque membre apporte aux organes de gouvernance» (Toé, 2014, p. 48).

Partant de là, Charreaux (1997) examine différents types de mécanismes participant à la gouvernance. Les «mécanismes externes intentionnels» incluent notamment les différents cadres législatifs auxquels l'entreprise doit se plier. Les «mécanismes 
externes spontanés» comprennent en particulier les conditions et normes établies par les marchés suivant lesquels les entreprises peuvent accéder aux ressources matérielles et financières nécessaires à leur développement. Les «mécanismes internes intentionnels" comprennent les différents organes de contrôles habituellement mis en place dans les entreprises, en particulier l'assemblée générale des actionnaires et le conseil d'administration. Les «mécanismes internes spontanés» relèvent de phénomènes plus informels et moins contrôlés tels que la culture d'entreprise dont les pressions normatives peuvent jouer un rôle déterminant sur les comportements des dirigeants. L'approche de la gouvernance proposée par Charreaux (1997) a ainsi permis d'élargir l'analyse en intégrant à la fois les propriétaires et les parties prenantes. Cette approche intègre ainsi des questions d'efficience et des questions de légitimité. Ce cadre d'analyse permet d'aborder la plupart des organisations, il a notamment été repris dans le champ des OBNL.

La gouvernance constitue un des axes de réflexion les plus importants de la recherche de ces dernières années sur les OBNL. Cette littérature s'interroge sur les spécificités de la gouvernance de ces organisations compte tenu d'un certain nombre de caractéristiques les différenciant des entreprises classiques. Boncler et Valéau (2010), Eynaud (2015), Hoarau et Laville (2008) se rejoignent ainsi sur deux spécificités des OBNL en lien avec la gouvernance : l'établissement du projet afin de donner un contenu au but non lucratif et à une mission souvent trop largement définie, et la notion de bien commun à défaut de propriété sur laquelle se fonde le principe "un homme, une voix». Suivant ce principe démocratique, la gouvernance ne concerne plus uniquement les initiatives des dirigeants élus, mais aussi celles des autres membres, ces derniers bénéficiant en général d'une large autonomie ainsi que d'un droit de regard sur les orientations stratégiques de leur OBNL. Cette littérature porte à la fois sur les mécanismes internes et externes : elle met en avant la capacité des OBNL à s'adapter à la compétition sur les «marchés» des subventions, des sponsorings ou des dons, tout en restant fidèles à leur mission et à leur culture d'origine.

Dans le cadre de cet article, nous focalisons notre attention sur les mécanismes internes intentionnels de la gouvernance des OBNL, autrement dit sur l'assemblée générale et le conseil d'administration. Les OBNL sont, comme les entreprises, gouvernées par une assemblée générale et un conseil d'administration, mais au lieu d'être réservées à des propriétaires actionnaires, ces deux instances sont, dans ce contexte, compte tenu de l'absence de propriété, composées de "membres» (Boncler \& Valéau, 2010). L'identification des parties prenantes reconnues comme membres est établie lors de la première assemblée et inscrite dans les statuts. Suivant les OBNL, ce statut de membre peut être ouvert à tous sur la base d'une simple adhésion ou contrôlé dans le cadre d'une procédure de cooptation plus restrictive (Boncler \& Valéau, 2010). Cette décision fondatrice détermine qui a le droit de vote lors des assemblées, qui peut être élu au CA et qui peut en devenir président. Comme le montrent Eynaud, Chatelain-Ponroy et Sponem (2015), la répartition des compétences et des pouvoirs entre l'assemblée, le conseil d'administration et le président varie considérablement d'une OBNL à l'autre. Dans le cadre de leur étude, ces auteurs identifient des cas où l'assemblée générale domine le conseil d'administration à travers ses débats, ses propositions et ses votes; des cas où ces deux instances coproduisent le projet; et enfin des cas où le projet est proposé par la CA à l'assemblée pour validation. Même dans ce dernier cas de figure, l’assemblée générale garde légalement le dernier mot, la plupart des législations nationales prévoyant au minimum une assemblée par an avec à l'ordre du jour le bilan de l'année passée, le projet pour l'année à venir et la mise au vote du renouvellement des mandats des dirigeants. Ces votes obligent les dirigeants élus à prendre en compte les points de vue des parties prenantes habilitées de façon à pouvoir constituer autour d'eux et de leur projet une majorité.

Les recherches de ces dernières années sur la gouvernance des OBNL ont surtout mis en exergue la "fonction cognitive» de l'assemblée générale et du conseil d'administration. Hoarau et Laville (2008) indiquent que dans les OBNL, la gouvernance partenariale et la gouvernance cognitive vont presque systématiquement de pair : les différents points de vue exprimés par les parties prenantes aident et incitent le président et les autres membres à prendre en compte différentes finalités et valeurs (Herman et Renz, 1997; Hoarau et Laville, 2008; Biondi et al., 2010). Cette idée a été notamment développée par Herman et Renz (1997) qui ont fait le lien entre diversité des parties prenantes et performance, en montrant qu'une gouvernance des OBNL élargie permet de prendre en compte toutes les dimensions d'une performance multicritère. Le modèle partenarial et cognitif offre ainsi une approche intégrative de la gouvernance des OBNL permettant de réunir autour du président l'ensemble des parties prenantes afin de développer le bien commun de façon efficiente et légitime. Valéau, Eynaud, Chatelain-Ponroy et Sponem (2018) ont récemment montré que la place accordée aux volontaires de terrain et aux usagers facilitait l'enracinement de la planification stratégique dans la mission, contribuant ainsi à la performance à la fois communautaire et économique de leur OBNL.

La «fonction disciplinaire» de l'assemblée générale est en revanche moins souvent abordée par la littérature comme par les acteurs eux-mêmes. Pourtant, comme le rappellent Boncler et Valéau (2010) cette dimension fait partie intégrante de ses prérogatives. L'assemblée générale a non seulement le pouvoir d'établir qui est membre et, ce faisant, qui peut participer à la définition du projet, et qui peut juger l'action des dirigeants et des autres membres sur cette base, mais elle possède aussi le pouvoir d'élire et de révoquer ces derniers. Ce pouvoir, juridiquement établi, est rappelé par le Haut Conseil à la Vie Associative : "une association peut souhaiter exclure un de ses membres pour un motif grave, par exemple des agissements préjudiciables aux intérêts de l'association, des conflits graves entre membres. [...] La procédure disciplinaire mise en place doit être celle définie par les statuts. En l'absence d'une instance spécialisée, l'assemblée générale joue ce rôle." (www.association.gouv.fr). Cette fonction disciplinaire était au cœur de l'article de Valéau (1999) sur l'autonomie conditionnelle accordée aux bénévoles. Cet auteur expliquait que cette fonction disciplinaire restait relativement taboue dans la mesure où elle ne cadre pas avec la sémantique fraternelle habituellement mise en avant par les OBNL. Valéau (1999) montrait ainsi que la liberté d'action et de parole des membres restait en pratique «conditionnelle» au respect des lignes directrices définies dans le cadre du projet. 
Notre étude ne porte pas directement sur l'exercice ordinaire de l'assemblée générale et du conseil d'administration, c'est-à-dire les discussions (cognitives) et les contrôles (disciplinaires) visant à confronter l'action des dirigeants et des autres membres au projet, mais sur la difficulté d'établir ce projet en cas de différends, voire de conflits, entre les parties prenantes habilitées à le définir. Cette perturbation se manifeste par une incapacité de l'assemblée générale et du conseil d'administration à établir un projet cohérent.

\section{LA GOUVERNANCE «DÉMOCRATIQUE» À L'ÉPREUVE DES DÉSACCORDS}

Alors que la plupart des textes sur la gouvernance des OBNL abordent la diversité des parties prenantes impliquées dans la gouvernance comme une source d'inspiration, de légitimité et d'efficacité, ce modèle se heurte à des réalités souvent ignorées de la vie des OBNL : celles des conflits. Ces conflits contredisent les synergies naturelles entre les différents modèles de la gouvernance.

L'origine de la plupart des conflits dans les OBNL découle de l'existence de deux projets différents susceptibles d'opposer entre eux les acteurs de la gouvernance (Drucker, 1990; Boncler \& Valéau, 2010; Valéau et al., 2013; Valéau, 2015). Ces conflits opposent souvent les parties prenantes de l'assemblée générale et les salariés (Boncler \& Valéau, 2010) ou les partenaires financiers (Valéau \& Annette, 2012), mais ils peuvent aussi survenir au sein même de l'assemblée entre les parties prenantes (Valéau et al., 2012). Ces tensions ont souvent pour objet des dilemmes entre respect absolu des valeurs et pragmatisme au service d'une plus grande efficacité (Laville, 2010). La méta-analyse de Valéau (2015) montre que presque toutes les OBNL étudiées par la littérature passent, à un moment de leur développement, par une crise liée à la confrontation entre une approche en termes d'efficience et une vision en termes de légitimité.

Au regard de la littérature, cette opposition entre les visions des différentes parties prenantes ne constitue pas une exception, mais une règle relativement fréquente, compte tenu de la nature duale des OBNL entre entreprise et communauté (Valéau, 2003; Rousseau, 2007; Reid \& Karambayya, 2009). En conséquence, la gouvernance des OBNL n'est pas forcément apaisée. Les cas étudiés par Kreutzer et Jager (2011) montrent les effets de ces conflits sur l'implication des acteurs. Mintzberg (1983) situe ces conflits dans le cadre d'une arène politique au sein de laquelle les acteurs perdent le sens de la mission. Ces désaccords au sommet peuvent menacer l'équilibre et le développement des OBNL.

La notion de désaccord met ainsi le modèle partenarial et cognitif, identifié comme fondement naturel de gouvernance démocratique des OBNL à l'épreuve de la réalité. Différents auteurs identifient ainsi certaines difficultés associées à la consultation régulière des parties prenantes (Valéau, 2003; Meier \& Schier, 2008). Certains auteurs évoquent la longueur des débats retardant l'action (Gawell, 2013; Hafsi \& Thomas, 2005). Comme le soulignent Meier et Schier (2008, p.20), une des principales limites de cette approche est «la multiplication d'objectifs hétérogènes difficiles à satisfaire et qui pose la question du contrôle de la conformité des actions de l'organisation à ces objectifs». Si l'agora de parties prenantes qui sous-tend le modèle partenarial et cognitif constitue un atout pour définir un projet légitime, prenant en compte les différents aspects liés à la mise en œuvre de la mission (Hoarau \& Laville, 2008; Herman \& Renz, 2008; Lecy et al., 2012), la consultation régulière multiplie les risques de désaccords ouverts (Mati, 2012; Valéau et al., 2013).

A l'inverse, d'autres auteurs évoquent un certain nombre de biais aboutissant à des réalisations tronquées du modèle partenarial. Différents auteurs pointent ainsi certaines difficultés voire certaines ambiguïtés dans la mise en œuvre du modèle partenarial : l'inclusion des uns et des autres dans les organes de gouvernance ne dépend pas seulement de leur légitimité, mais aussi de leur pouvoir et de l'urgence perçue de leurs droits (Mitchell, Agle \& Wood, 1997; Bouglet \& Joffre, 2015). Les OBNL intègrent volontairement un certain nombre d'acteurs en tant que parties prenantes (El Abboubi, 2013; Bouglet \& Joffre, 2015). El Abboubi (2013) souligne le caractère socialement construit du statut de «partie prenante». Certaines parties prenantes peuvent ainsi être écartées par négligence ou de façon plus délibérée, à des fins d'efficience.

Nous retrouvons, à travers les deux pratiques de la gouvernance démocratique dans les OBNL ci-dessus évoquées, un dilemme semblable à celui identifié par les sciences politiques au sein des sociétés démocratiques contemporaines entre des formes de démocratie dites "représentatives» et des formes dites «participatives» (Blondiaux, 2008; Callon et al., 2001; Koeble, 2006). La gouvernance démocratique des OBNL, sur le principe "un homme - une voix", apparaît, suivant les interprétations de Hoarau et Laville (2008) des grilles de Charreaux (2003), fondamentalement partenariale et cognitive, mais elle peut, en pratique, suivant la grille des sciences politiques, être opérationnalisée sous des formes «représentatives» ou "participatives». La démocratie "représentative» apparaît comme efficiente car elle permet des suites de décisions rapides et cohérentes, mais elle pose un problème de légitimité dans la mesure où elle n'implique que très ponctuellement des citoyens se reconnaissant de moins en moins dans ce mode de fonctionnement (Blondiaux, 2008). Les formes de démocratie dites «participatives» ont pour objectif de raviver les démocraties modernes en réinstaurant des espaces publics (Habermas, 1962) dans le cadre desquels les citoyens peuvent débattre, délibérer et participer à la prise de décision. Elles apparaissent cependant, selon Blondiaux (2008), difficiles à mettre en pratique. Dans les $\mathrm{OBNL}$, suivant un modèle démocratique dit "représentatif», le président privilégierait une exécution efficiente du projet sur la base duquel il a été élu, autrement dit, il tenterait de réaliser l'«idéal du projet». Cette approche n'est pas sans rappeler la démarche entrepreneuriale telle que définie par Filion (1990), celle d'un individu porteur d'une vision stratégique cherchant à la concrétiser de la façon la plus efficiente possible. Suivant une approche démocratique dite "participative», le président multiplierait les consultations et les délibérations collectives afin de permettre à l'ensemble des parties prenantes de négocier, de résoudre leurs différends à travers l'élaboration conjointe d'un projet reconnu, légitimé par tous, autrement dit, il prioriserait «un consensus idéal».

Dans le cadre de cette recherche empirico-formelle, nous explorons les désaccords comme des incidents critiques révélateurs d'arbitrages souvent implicites effectués par le président entre les fonction cognitive et disciplinaire de l'assemblée. Face à ces situations de désaccord, les présidents se trouvent souvent pris au dépourvu et s'interrogent sur la conduite à tenir. Dans 
le cadre de cette recherche, nous nous interrogeons cependant sur la place du président : s'il apparaît normal et légitime que les différentes parties prenantes défendent leurs convictions, qu'en est-il du président? Peut-il être un acteur comme les autres ou peut-il se situer au-dessus des parties? Autrement dit, doit-il utiliser son pouvoir pour défendre ses propres convictions ou doit-il, après avoir tenté de convaincre, lâcher prise sur ces dernières afin de devenir le garant d'un processus de co-construction visant le consensus? Il appartient alors au président de restaurer les mécanismes internes intentionnels supposés réguler son action et celles des autres acteurs, en optant soit pour l'idéal du projet (le sien), soit pour l'idéal démocratique suivant lequel il adapte sa vision en en fonction des demandes portées par les différentes parties prenantes. Le sujet n'est pas la gouvernance en tant que tel mais les restaurations des mécanismes de la gouvernance mis à mal par les conflits.

\section{Méthodologie qualitative}

Ces questions de gouvernance sont, selon nous, au cœur des sciences de gestion appliquées au monde des OBNL. Elle détermine une grande partie du développement technico-économique et socio-politique de ces structures. Il convient ainsi de garder un point de vue purement scientifique et d'éviter les positions normatives (Laville, 2010; Eynaud, 2015). C'est pourquoi nous «ancrons» notre recherche (Glaser \& Strauss, 1967) dans les expériences vécues des présidents, en lien avec les décisions et pratiques qui sont les leurs. Nous examinons leurs positionnements mais aussi les processus d'apprentissage sur lesquels ils se fondent.

L'objectif de cette recherche était d'étudier l'expérience vécue et les décisions prises par les présidents depuis leur prise de fonction. L'entretien semi-directif nous est apparu comme l'outil le plus adapté pour pouvoir opérer ce type de rétrospective. Nous avons effectué un total de 30 entretiens. Les 22 entretiens principaux ont été effectués avec les présidents eux-mêmes. Ces derniers appartenaient à des organisations de différentes tailles (entre 0 et 260 salariés; de 1000 à 3,6 millions d'euros de budget annuel) et d'anciennetés variées (de 1 à 42 années d'existence), intervenant dans différents secteurs d'activités (insertion, éducation populaire, sport, culture, protection de l'environnement). En complément de ces entretiens principaux, nous avons également pu, dans un certain nombre de cas, interroger d'autres acteurs : nous avons effectué des entretiens avec trois directeurs et cinq membres du CA. Ces données auxiliaires constituent une forme de triangulation des sources (Denzin et Lincoln, 1994). Deux des chercheurs étaient également en situation d'observation participante, l'un en tant que directeur, l'autre en tant que président, ce qui nous a permis des interprétations croisées des situations rapportées dans le cadre des entretiens. Ces entretiens ont été effectués en France au cours de ces trois dernières années, mais les témoignages ainsi recueillis reviennent sur des évènements et changements se déroulant sur des périodes remontant parfois jusqu'à 20 ans en arrière. Ces deux dernières décennies ont été marquées par une montée des exigences en termes de professionnalisation, ce qui s'est traduit par de nombreuses tensions autour, mais aussi à l'intérieur même de ces organisations comme le montrent Valéau et al. (2012). Cette période explique sans doute partiellement les différends fréquents relevés dans le cadre de nos données.
Nos entretiens visaient à aborder avec nos interlocuteurs les différents éléments de notre problématique, notre grille d'entretien comprenait ainsi les points suivants : les grands dilemmes rencontrés au cours des années passées, les points de vue et arguments exprimés par les parties prenantes présentes au sein de l'AG, les différends, les décisions prises par le président pour réguler ces derniers et les rationalités sous-jacentes en termes de légitimité ou d'efficience, le retour à la normal. Dans le cadre des entretiens avec les présidents, après avoir demandé à la personne interviewée de se présenter et de présenter son OBNL, nos entretiens démarraient avec la question suivante: «quels ont été les grands moments, les grandes décisions qui ont marqué votre parcours au sein de cette $O B N L$ ?». Cette question se voulait délibérément plus large que notre problématique et que notre guide d'entretien pour deux raisons : pour ne pas induire de réponses a priori et pour conserver une semi-directivité ouverte à la découverte (Fontana \& Frey, 1994). Les «grands moments » correspondaient généralement à des grands débats ayant marqué l'OBNL, les décisions prises par le président intégrait généralement soit une visée consensuelle, soit une recherche d'efficience dans la réalisation de leur propre vision du projet. Dans la plupart des cas, la personne interviewée abordait spontanément l'ensemble des points constituant notre grille d'entretien, même si ce n'était pas forcément dans l'ordre. Nous cochions pendant l'entretien ces points au fur et à mesure sur notre grille. Seulement si la personne n'avait pas abordé spontanément un des points, nous posions des questions plus explicites, par exemple : "avez-vous dû, déjà, faire face à des désaccords au sein de l'assemblée?».

Suivant une approche empirico-formelle (Glaser \& Strauss, 1967; Denzin \& Lincoln, 1994), le traitement des données s'est fait sous la forme de codages itératifs émergeant d'allers-retours entre terrain et théories. Une première codification «in vivo", consistant à résumer les propos des présidents et des autres acteurs, est présentée dans la première colonne des tableaux de la section suivante. Suivant les traditions abductives (Dubois \& La Gadde, 2002), les codes finaux présentés dans la figure 1 résultent d'une longue série d'allers-retours entre les grilles d'analyse théoriques présentées dans leur version finale dans la première partie de cet article et les contenus des retranscriptions des entretiens. Il peut être utile d'évoquer certains des cheminements ainsi suivis (Valéau \& Gardody, 2015). Concernant la gouvernance, nous étions partis des modèles établis par Charreaux (2003), avec notamment le modèle actionnarial, partenarial et cognitif, avant de constater la difficulté de les appliquer stricto sensu aux OBNL. Nous sommes alors revenus aux mécanismes internes et externes, intentionnels et spontanés ayant une portée à la fois disciplinaire et cognitive qui sous-tendent ces modèles (Charreaux, 1997). Nous avons ainsi centré nos réflexions sur la capacité de l'assemblée générale et du conseil d'administration, du fait de leur composition, à produire un projet ayant une fonction à la fois cognitive et disciplinaire. L'étape suivante a consisté à identifier les différends comme une forme de détérioration de ces mécanismes et une forme de paradoxe suivant lequel le président est lui-même amené à restaurer ces mécanismes en vertu desquels son action sera régulée. Cette démarche est d'autant plus ambiguë qu'elle remet en question la possibilité d'émergence d'une opposition, voire d'une nouvelle coalition, en posture de diriger. Ces questions liées à la 
gouvernance démocratique des OBNL nous ont conduit à des emprunts du côté des sciences politiques. Nous avons ainsi identifié deux orientations de la gouvernance des OBNL : l'une visant l'efficience à travers des démarches entrepreneuriales dans le cadre d'une version représentative de la gouvernance démocratique; l'autre visant la légitimité à travers une approche communautaire dans le cadre d'une version participative de la gouvernance démocratique.

\section{Résultats}

Nous commençons la présentation de nos résultats en montrant le basculement de situations caractérisées par une assemblée et un conseil efficacement et une assemblée et un conseil efficacement et légitimement unis autour d'un même projet à des situations de désaccords. Ces derniers amènent les présidents à constater l'impossibilité de satisfaire simultanément l'idéal du projet et l'idéal consensuel, autrement dit à maintenir conjointement les fonctions cognitive et disciplinaire de l'assemblée et du conseil. Face à ces difficultés, deux grandes voies de résolution émergent de nos données :

- la première privilégie l'idéal du projet dans le cadre d'une gouvernance démocratique de type représentatif privilégiant la fonction disciplinaire de l'assemblée et du conseil d'administration;

- la seconde, relativement moins fréquente au regard de nos données, donne priorité à cet idéal de démocratie qu'est le consensus, dans le cadre d'une gouvernance démocratique de type participatif visant à maintenir la fonction cognitive de ces deux instances.

\section{LES DIFFÉRENDS OU L'IMPOSSIBILITÉ DU DOUBLE IDÉAL}

Cette sous-partie revient sur les expériences du différend rapportées par les présidents et les autres acteurs rencontrés. Ces différends posent une difficulté voire une impossibilité de maintenir l'orientation du projet tout en conservant le consensus de départ. Cette volonté de départ correspondant dans le cadre de la figure 1 à la position « $50 \%-50 \%$ » consistant à accorder une importance égale à ces deux approches de la gouvernance des OBNL. Nous codons ces différends en termes de «désaccord» lorsque les parties-prenantes expriment des points de vue différents, et en termes de "conflits» lorsque les échanges se chargent d'émotions négatives.

Comme le montre le premier extrait du tableau 1, la vie des OBNL commence souvent dans la plus grande cordialité. Les échanges sont constructifs et respectueux et contribuent au développement d'une action collective au service d'un projet partagé par tous. La notion de «choc» ici évoquée traduit à la fois la surprise et la déception lorsqu'apparaît le premier désaccord (extrait 1.8). Les présidents et ceux qui les entourent font ainsi face à un décalage entre leur idéal d'action collective au service d'un projet et la réalité du terrain, d'autant que le désaccord risque de tourner au conflit.

Comme le montrent les extraits 1.2, 1.3 et 1.4, les désaccords et conflits au sein des OBNL portent sur les finalités, mais aussi, très souvent, sur les moyens et le fonctionnement, une même fin pouvant justifier différents moyens. Ces trois extraits évoquent la confrontation classique entre les tenants d'un entrepreneuriat envisageant un professionnalisme formalisé comme un moyen adéquat et les défenseurs d'une communauté plus spontanée gardant des liens sociaux plus primaires. Dans les deux cas, les acteurs se manifestent pour dénoncer ce qu'ils perçoivent comme une dérive. Cependant, le désaccord résulte surtout dans la manière d'échanger ces points de vue. Les extraits 1.2 à 1.4 montrent une escalade dans les modes d'expression. L'extrait 1.2 constitue une façon relativement neutre de «tirer la sonnette d'alarme». Dans l'extrait 1.3, cet ancien chef d'entreprise ne remet pas intrinsèquement en cause l'OBNL, mais indique ne pas s'être adapté à son fonctionnement communautaire fondé sur une grande part d'informel. L'extrait 1.4 introduit une posture plus radicale : les acteurs mettent «les pieds dans le plat ", ils déclenchent un état d'urgence de façon plus offensive. Le conflit devient alors plus ouvert (extrait 1.5), les individus se retirent (extraits 1.6 et 1.7). Le fonctionnement «idéal» de l'organisation s'en retrouve perturbé, l'OBNL est fragilisée.

FIGURE 1

Codages

\begin{tabular}{|c|c|c|}
\hline Mise en œuvre, finalité sociale & Entrepreneuriat & \\
\hline Approche disciplinaire & Représentation & \\
\hline Débat, appartenance & Communauté & \multirow{2}{*}{ Légitimité } \\
\hline Approche cognitive & Participation & \\
\hline Expression, objection & Désaccord & \multirow{2}{*}{ Différends } \\
\hline Contestation, démission & Conflits & \\
\hline Optimisation projet / consensus & Idéal du projet & \multirow{3}{*}{ Arbitrage } \\
\hline Instabilité, confusion, doute & $50 \% 50 \%$ & \\
\hline Optimisation consensus / projet & Consensus idéal & \\
\hline
\end{tabular}

1. Cette présentation se réfère aux nouvelles normes des recherches qualitatives établies dans la revue Academy of Management Journal. 
Le premier apprentissage des présidents dans le cadre de la gouvernance des OBNL est que la réalité n'est pas aussi simple et idyllique qu'ils ne l'avaient anticipée (extrait 1.8). Ils comprennent ainsi que l'harmonie des premiers mois ou des premières années ne peut être tenue pour acquise. Les désaccords tournent souvent aux conflits et sont vécus par les acteurs comme des échecs. Face aux dysfonctionnements qui en découlent, des solutions doivent être trouvées.

\section{LA PRIORITÉ DONNÉE À L'IDÉAL DU PROJET ET À LA FONCTION DISCIPLINAIRE}

Une partie des acteurs rencontrés considèrent que l'action collective n'est possible que si les valeurs de base sur lesquelles se fonde le projet sont partagées par tous. Cette approche du fonctionnement des OBNL pose, ce faisant, certaines limites de la démocratie et définit un périmètre au-delà duquel cette dernière ne s'applique plus complètement. Da façon surprenante, suivant cette approche le contrôle disciplinaire s'exerce sur la minorité en désaccord. La neutralisation ou l'exclusion, directe ou indirecte, des acteurs contestataires, est présentée comme une mesure nécessaire en vue de préserver l'OBNL et son projet. Nous codons ces positionnements en termes d'«entrepreneuriat» pour désigner les registres justifiant de ces approches pragmatiques de l'action des OBNL et de "gouvernance démocratique représentative» pour identifier les rôles joués par le président dans le cadre des actions ainsi mises en œuvre à cette fin (tableau 2).

Les trois premiers extraits du tableau 2 évoquent un « entrepreneuriat" porté par une vision qu'il s'agit de réaliser de la façon la plus efficiente possible. Ils reprennent, ce faisant, certains fondements traditionnels du mouvement des OBNL avec une affirmation des valeurs inspirant le projet (extraits 2.1, 2.2 et 2.3). En même temps qu'un certain pragmatisme, ces discours expriment ainsi un fort engagement militant fondé sur des convictions humanistes. Cependant, les acteurs s'exprimant dans l'extrait 2.2 et, de façon plus marquée, dans l'extrait 2.3 introduisent ce qu'ils considèrent comme une condition nécessaire à ce fonctionnement : les membres de l'OBNL doivent, selon eux, adhérer à ce projet et ces valeurs. En cas de désaccords

\begin{tabular}{|c|c|c|c|}
\hline \multicolumn{4}{|c|}{$\begin{array}{l}\text { TABLEAU } 1 \\
\text { Les différends }\end{array}$} \\
\hline Réf. & Codes & Sources $\&$ contextes & Extraits \\
\hline 1.1. & $\begin{array}{l}\text { Désaccord (absencel } \\
\text { - Une réalité initialement } \\
\text { conforme au double idéal }\end{array}$ & $\begin{array}{l}\text { Président } \\
+ \text { de } 10 \text { ans d'ancienneté } \\
\text { + de } 10 \text { salariés } \\
\text { Insertion }\end{array}$ & $\begin{array}{l}\text { "Je crois que la décision se prenait de façon collégiale parce qu'on } \\
\text { n'avait pas eu de désaccord jusque-là. C'était assez évident, on discutait } \\
\text { ensemble et puis il y avait une espèce de décision qui s'imposait. On } \\
\text { prenait le temps. On a passé de bons moments.» }\end{array}$ \\
\hline 1.2 & $\begin{array}{l}\text { Désaccord } \\
\text { - Désaccord sur le } \\
\text { fonctionnement. } \\
\text { - Tension entre le CA et les } \\
\text { membres de base concernant } \\
\text { les prises de décision }\end{array}$ & $\begin{array}{l}\text { Membre bureau } \\
+ \text { de } 10 \text { ans d'ancienneté } \\
+ \text { de } 10 \text { salariés } \\
\text { Insertion }\end{array}$ & $\begin{array}{l}\text { «Ma question est : sommes-nous dans cette OBNL dans un système } \\
\text { pyramidal où le bureau en haut ne fait que de la «stratégie» et en } \\
\text { bas on délègue tout aux «petits» employés et bénévoles (méthode } \\
\text { de l'entreprisel ou bien sommes-nous une vraie OBNL où chaque } \\
\text { membre est sur un pied d'égalité et participe à chaque opération?» }\end{array}$ \\
\hline 1.3 & $\begin{array}{l}\text { Désaccord } \\
\text { - L'expression des désaccords } \\
\text { concernant le fonctionnement }\end{array}$ & $\begin{array}{l}\text { Président } \\
+ \text { de } 10 \text { ans d'ancienneté } \\
+ \text { de } 10 \text { salariés } \\
\text { Insertion }\end{array}$ & $\begin{array}{l}\text { "ll a dit honnêtement : vous marchez de manière familiale, je } \\
\text { comprends, ça ne me gêne pas, ça a l'air de fonctionner, mais moi je } \\
\text { ne peux pas fonctionner commeça.» }\end{array}$ \\
\hline 1.4 & $\begin{array}{l}\text { Désaccord et Conflit } \\
\text { - Objection, contestation, } \\
\text { opposition }\end{array}$ & $\begin{array}{l}\text { Président } \\
\text { - de } 10 \text { ans d'ancienneté } \\
\text { - de } 10 \text { salariés } \\
\text { Education populaire }\end{array}$ & $\begin{array}{l}\text { «Le nouveau trésorier trouvait que ça n'allait pas comme ça. Il a mis } \\
\text { les pieds dans le plat.» }\end{array}$ \\
\hline 1.5 & $\begin{array}{l}\text { Conflit } \\
\text { - Cercle vicieux du différend au } \\
\text { conflit } \\
\text { - Emotions négatives et } \\
\text { souffrance }\end{array}$ & $\begin{array}{l}\text { Président } \\
\text { - de } 10 \text { ans d'ancienneté } \\
\text { - de } 10 \text { salariés } \\
\text { Insertion }\end{array}$ & $\begin{array}{l}\text { "Après, il y a eu une période où s'est allé en se dégradant de plus en } \\
\text { plus...C'était absolument épouvantable.» }\end{array}$ \\
\hline 1.6 & $\begin{array}{l}\text { Conflit } \\
- \text { Un fonctionnement perturbé }\end{array}$ & $\begin{array}{l}\text { Président } \\
\text { - de } 10 \text { ans d'ancienneté } \\
\text { - de } 10 \text { salariés } \\
\text { Insertion }\end{array}$ & $\begin{array}{l}\text { "L'impact est le blocage des projets, la frustration des membres du } \\
\text { conseil d'administration, la non-adhésion des employés aux décisions.» }\end{array}$ \\
\hline 1.7 & $\begin{array}{l}\text { Conflit } \\
\text { - Des associations fragilisées }\end{array}$ & $\begin{array}{l}\text { Président } \\
\text { + de } 10 \text { ans d'ancienneté } \\
\text { - de } 10 \text { salariés } \\
\text { Insertion }\end{array}$ & $\begin{array}{l}\text { "Des démissions successives au sein de l'association ont fragilisé } \\
\text { les relations entre le CA et les salariés.» }\end{array}$ \\
\hline 1.8 & $\begin{array}{l}\text { Conflit } \\
- \text { Une déception }\end{array}$ & $\begin{array}{l}\text { Président } \\
+ \text { de } 10 \text { ans d'ancienneté } \\
\text { - de } 10 \text { salariés } \\
\text { Insertion }\end{array}$ & $\begin{array}{l}\text { "Je suis trop déçu par ce manque de cohésion des piliers de } \\
\text { l'association. Alors je continue les demandes de subvention.» }\end{array}$ \\
\hline
\end{tabular}

Note : les extraits d’entretiens ci-dessus évoquent les relations entre le président et l'assemblée générale à travers les différents moments dans la vie de l'association : dans le cadre de réunions en AG ordinaire ou extraordinaire, en conseil d'administration lémanation restreinte de l'AG) mais aussi lors de confrontations plus informelles. 
(cf. tableau 1), lorsque cette condition n'est plus remplie, l'affectif et la démocratie peuvent être momentanément suspendus (extraits 2.3, 2.4 et 2.7). Les présidents et les acteurs de cette coalition déclenchent une forme d'état d'urgence dans le cadre duquel d'autres pratiques sont exceptionnellement mises en place : recadrage (extrait 2.6), mais aussi exclusion (extrait 2.7). Bien sûr, certaines de ces prises de pouvoir peu démocratiques peuvent être dénoncées, mais elles restent, selon ces acteurs, exceptionnelles et momentanées. Les OBNL reviennent ensuite à un fonctionnement démocratique (extrait 2.9), la gouvernance partenariale reprend alors ses droits.

L'apprentissage et la construction socialement élaborée consistent ici à fixer les limites et les conditions de l'exercice démocratique nécessaire au maintien du projet. Le choc se manifeste par la transgression de l'idéal consensuel, voire de l'idéal démocratique initialement posé. La manière dont s'opère cette prise de pouvoir varie mais, compte tenu du blocage démocratique, les OBNL ainsi gouvernées passent par une loi d'exception permettant des jeux de pouvoir parfois relativement durs, avant de revenir à une fonction démocratique. La légitimité de ces actions relatives aux minorités se fonde sur la majorité, mais aussi sur le mandat exécutif confié au président suite à son élection.

\section{LA PRIORITÉ DONNÉE À L'IDÉAL CONSENSUEL ET À LA FONCTION COGNITIVE DE LA GOUVERNANCE}

Le choix de la démocratie souvent annoncé dans les discours, se pose de façon plus problématique dans la pratique. Il ne s'agit pas ici, comme dans le cas précédent, d’une démocratie représentative, mais d'une démocratie plus absolue fondée sur une participation plus fréquente et plus poussée des parties prenantes dans la construction du projet. Concrètement, cela signifie que les présidents et leurs équipes, bien qu'à priori en situation de contrôle, acceptent, au nom de la légitimité et du consensus, de perdre en partie le contrôle du projet, en remettant régulièrement son orientation aux votes des parties prenantes en présence.

Le premier extrait du tableau 3 pose une vision idyllique, mais finalement assez peu fréquente de l'exercice démocratique

\begin{tabular}{|c|c|c|c|}
\hline \multicolumn{4}{|c|}{$\begin{array}{l}\text { TABLEAU } 2 \\
\text { Priorité au projet }\end{array}$} \\
\hline Réf. & Codes & Source $\&$ contexte & Extraits \\
\hline 2.1 & $\begin{array}{l}\text { Discipline } \\
\text { Mise en œuvre }\end{array}$ & $\begin{array}{l}\text { Président } \\
+ \text { de } 10 \text { ans d'ancienneté } \\
+ \text { de } 10 \text { salariés } \\
\text { Environnement }\end{array}$ & $\begin{array}{l}\text { «On était très au clair dans notre projet, dans notre diversité. Les } \\
\text { valeurs étaient bien établies. On n'a jamais eu aucun doute au niveau } \\
\text { du projet. Les fondamentaux étaient là. Ce qu'on voulait, c'était pouvoir } \\
\text { le mettre en œuvre. » }\end{array}$ \\
\hline 2.2 & $\begin{array}{l}\text { Discipline } \\
\text { Président porteur du projet }\end{array}$ & $\begin{array}{l}\text { Membre CA } \\
+ \text { de } 10 \text { ans d'ancienneté } \\
+ \text { de } 10 \text { salariés } \\
\text { Insertion }\end{array}$ & $\begin{array}{l}\text { «ll y a une relation de confiance étroite parce que le président est le } \\
\text { responsable civil, pénal. A partir de là, s'ilya, ne serait-ce qu'un doute, } \\
\text { ça ne peut pas fonctionner.» }\end{array}$ \\
\hline 2.3 . & $\begin{array}{l}\text { Discipline \& optimisation } \\
\text { du projet } \\
\text { La nécessité de défendre } \\
\text { le projet }\end{array}$ & $\begin{array}{l}\text { Président } \\
+ \text { de } 10 \text { ans d'ancienneté } \\
+ \text { de } 10 \text { salariés } \\
\text { Insertion }\end{array}$ & $\begin{array}{l}\text { "Notre vision sociale est simple : partager sans oublier de favoriser les } \\
\text { plus démunis. Notre vision économique est pragmatique : sans une saine } \\
\text { gestion rien n'est durable. (...) Nous avons été contraints d'éloigner des } \\
\text { instances de décision certains membres à l'approche trop mercantile.» }\end{array}$ \\
\hline 2.4 & $\begin{array}{l}\text { Discipline \& optimisation } \\
\text { du projet } \\
\text { Une absence momentanée } \\
\text { d'affectif }\end{array}$ & $\begin{array}{l}\text { Président } \\
+ \text { de } 10 \text { ans d'ancienneté } \\
\text { - de } 10 \text { salariés } \\
\text { Insertion }\end{array}$ & «Moi, j'en avais marre mais à l'époque, j'avais retiré l'affectif.» \\
\hline 2.5 & $\begin{array}{l}\text { Discipline, optimisation du } \\
\text { projet et légitimité labsence) } \\
\text { Une absence momentanée de } \\
\text { démocratie }\end{array}$ & $\begin{array}{l}\text { Membre CA } \\
+ \text { de } 10 \text { ans d'ancienneté } \\
\text { - de } 10 \text { salariés } \\
\text { Education populaire }\end{array}$ & $\begin{array}{l}\text { "La prise de décision n'est pas démocratique, les autres membres du } \\
\text { bureau sont obligés de subir les conséquences.» }\end{array}$ \\
\hline 2.6 & $\begin{array}{l}\text { Discipline et légitimité } \\
\text { labsencel } \\
\text { Les recadrages }\end{array}$ & $\begin{array}{l}\text { Président } \\
+ \text { de } 10 \text { ans d'ancienneté } \\
+ \text { de } 10 \text { salariés } \\
\text { Insertion }\end{array}$ & $\begin{array}{l}\text { «Il nous disait, «vous ne comprenez rien». En fait, chaque fois il revenait } \\
\text { avec cette logique-là, à chaque fois on le repositionnait.» }\end{array}$ \\
\hline 2.7 & $\begin{array}{l}\text { Discipline \& optimisation } \\
\text { du projet } \\
\text { L'exclusion des bénévoles }\end{array}$ & $\begin{array}{l}\text { Membre CA } \\
+ \text { de } 10 \text { ans d'ancienneté } \\
+ \text { de } 10 \text { salariés } \\
\text { Insertion }\end{array}$ & $\begin{array}{l}\text { «ll est déjà arrivé que certains ne soient pas d'accord. Dans ce cas, on } \\
\text { essaie de les pousser à la sortie.» }\end{array}$ \\
\hline 2.8 & $\begin{array}{l}\text { Discipline \& légitimité } \\
\text { labsencel } \\
\text { Des démissions }\end{array}$ & $\begin{array}{l}\text { Membre bureau } \\
+ \text { de } 10 \text { ans d'ancienneté } \\
+ \text { de } 10 \text { salariés } \\
\text { Education populaire }\end{array}$ & $\begin{array}{l}\text { «Personnellement, vu le manque d>engagement du bureau dans les } \\
\text { opérations, je vous annonce que je ne m>occuperai plus de cette activité } \\
\text { ni de l>organisation draucun événement lié à cette activité.» }\end{array}$ \\
\hline 2.9 & $\begin{array}{l}\text { Discipline } \\
\text { Le retour à la normale }\end{array}$ & $\begin{array}{l}\text { Président } \\
+ \text { de } 10 \text { ans d'ancienneté } \\
+ \text { de } 10 \text { salariés } \\
\text { Culture }\end{array}$ & «La gouvernance de l'OBNL est passée par plusieurs cycles de conflits.» \\
\hline
\end{tabular}

Note : les extraits d'entretiens ci-dessus évoquent les relations entre le président et l'assemblée générale à travers les différents moments dans la vie de l'association : dans le cadre de réunions en AG ordinaire ou extraordinaire, en conseil d'administration (émanation restreinte de l'AG) mais aussi lors de confrontations plus informelles. 
en OBNL. En pratique, la gouvernance se concrétise souvent par l'exercice du vote à la majorité (extrait 3.2). Suivant une version plus élaborée, certaines OBNL tentent cependant de pousser plus loin le débat (extraits 3.3, 3.4 et 3.5). Cependant, ces débats peuvent tourner à la controverse permanente où tout peut être discuté sans fin (extrait 3.4). Finalement, la plupart des OBNL finissent par trouver le bon usage, un usage opérationnel de ce fonctionnement partenarial dans le cadre duquel les acteurs apprennent à séparer le temps du débat contradictoire et le temps d'une convergence nécessaire pour pouvoir parvenir à une décision (extraits 3.5).

L'apprentissage ne concerne pas uniquement la construction d'usage régulant le processus décisionnel, le président et son équipe doivent également apprendre à lâcher prise et à faire des concessions sur le projet. En effet, ces confrontations démocratiques autour d'un projet apparaissent relativement indéterminées (extraits 3.2 et 3.5). Le président et son équipe renoncent ainsi à contrôler la décision pour devenir les garants d'un processus démocratique : ils s'assurent que la diversité des parties prenantes est représentée, et que ces derniers s'expriment et contribuent à la décision finale (extraits 3.6 et 3.7).

\section{Discussion}

Il convient tout d'abord de rappeler que le but de cette recherche n'était pas de cautionner l'une ou l'autre de ces deux approches de la gouvernance démocratique des OBNL, mais simplement d'examiner la façon dont elles sont portées et justifiées par les acteurs eux-mêmes. L'objectif de notre recherche était de comprendre comment les acteurs accédant à la fonction de président confrontent le double idéal des OBNL, c'est-à-dire l'idéal du projet et l'idéal consensuel. Comme le rappelaient nos premiers résultats (tableau 1), les différentes parties prenantes participant à l'élection portent souvent différentes visions du projet et différentes conceptions de gouvernance des OBNL. Ce faisant, les désaccords et les controverses restent potentiellement au cœur de la gouvernance de ces organisations (Valéau, 2003, 2015).

Nous identifions deux voies pour résoudre ces désaccords, la voie disciplinaire donnant la primauté au projet sur le consensus (figure 2.C - $75 \%$ projet) et la voie cognitive accordant la priorité au consensus en renonçant complètement ou en partie à contrôler le projet (figure 2.D - $75 \%$ consensus). L'arbitrage entre ces deux voies constitue un apprentissage consistant à accepter

\begin{tabular}{|c|c|c|c|}
\hline \multicolumn{4}{|c|}{$\begin{array}{l}\text { TABLEAU } 3 \\
\text { Priorité au consensus }\end{array}$} \\
\hline Réf. & Codages & Source \& contexte & Extraits \\
\hline 3.1 & $\begin{array}{l}\text { Cognition \& légitimité } \\
\text { Débat }\end{array}$ & $\begin{array}{l}\text { Membre bureau } \\
+ \text { de } 10 \text { ans d'ancienneté } \\
+ \text { de } 10 \text { salariés }\end{array}$ & $\begin{array}{l}\text { "C'est un peu ça l'intérêt de fonctionner à plusieurs, c'est d'objectiver. De } \\
\text { ne pas en faire une affaire perso. Déjà, on en sort grandi soi-même. (...) Et ça } \\
\text { arrive de temps en temps, en bureau, on arrive avec une idée, on ressort avec } \\
\text { une idée différente.» }\end{array}$ \\
\hline 3.2 & $\begin{array}{l}\text { Cognition, légitimité } \\
\text { \& optimisation } \\
\text { consensus } \\
\text { Débat et vote }\end{array}$ & $\begin{array}{l}\text { Membre bureau } \\
+ \text { de } 10 \text { ans d'ancienneté } \\
\text { - de } 10 \text { salariés }\end{array}$ & $\begin{array}{l}\text { "C'est par vote du conseil d'administration de ce qui est à l'ordre du jour. Le } \\
\text { président établit l'ordre du jour. (..) Le président ne prend jamais de décisions } \\
\text { seul. Il demande l'avis de tout le monde. On discute. Il y en a qui sont d'accord. } \\
\text { D'autres ne le sont pas. Et après, on fait un vote à main levée pour voir qui est } \\
\text { d'accord sur tel ou tel projet. Suivant le nombre de votes, soit le projet passe, } \\
\text { soit il ne passe pas.» }\end{array}$ \\
\hline 3.3 & $\begin{array}{l}\text { Cognition, légitimité \& } \\
\text { idéal du consensus } \\
\text { Débat et recherche du } \\
\text { compromis }\end{array}$ & $\begin{array}{l}\text { Président } \\
+ \text { de } 10 \text { ans d'ancienneté } \\
+ \text { de } 10 \text { salariés }\end{array}$ & $\begin{array}{l}\text { "S'il y a quelqu'un qui est contre, on n'a jamais d'imposition de l'opinion } \\
\text { majoritaire. (...) On a toujours essayé de trouver un juste milieu et un compromis, } \\
\text { alors que d'autres associations auraient dit on est cinq, le sixième est contre. } \\
\text { La majorité l'emporte.» }\end{array}$ \\
\hline 3.4 . & $\begin{array}{l}\text { Cognition \& légitimité } \\
\text { Du débat à la } \\
\text { controverse }\end{array}$ & $\begin{array}{l}\text { Président } \\
+ \text { de } 10 \text { ans d'ancienneté } \\
\text { - de } 10 \text { salariés }\end{array}$ & $\begin{array}{l}\text { "On évolue dans la controverse. Sans se dire qu'on va se bagarrer. C'est épuisant } \\
\text { car on a autre chose à faire dans la vie. C'est dans ces situations qu'on apprend } \\
\text { le plus.» }\end{array}$ \\
\hline 3.5 & $\begin{array}{l}\text { Cognition, légitimité } \\
\text { \& optimisation } \\
\text { consensus } \\
\text { Des usages de la } \\
\text { démocratie }\end{array}$ & $\begin{array}{l}\text { Membre bureau } \\
+ \text { de } 10 \text { ans d'ancienneté } \\
+ \text { de } 10 \text { salariés }\end{array}$ & $\begin{array}{l}\text { "Chaque fois que ça remonte en conseil d'administration, on commence à } \\
\text { retourner les trucs, à peser le pour et le contre. On a une manière de reposer } \\
\text { les problèmes qui est devenue une habitude.» }\end{array}$ \\
\hline 3.6 & $\begin{array}{l}\text { Cognition, légitimité } \\
\text { labsence) \& } \\
\text { consensus idéal } \\
\text { Promouvoir le débat }\end{array}$ & $\begin{array}{l}\text { Président } \\
+ \text { de } 10 \text { ans d'ancienneté } \\
+ \text { de } 10 \text { salariés }\end{array}$ & $\begin{array}{l}\text { "Ça m'embêtait qu'il n'y ait pas plus d'opposition. C'est pour cela que j'ai fait } \\
\text { venir d'autres gens aussi. Finalement, c'était une position qui était confortable } \\
\text { mais que je n'aimais pas. J'essayais. Je voulais que ça discute mais on était } \\
\text { trop d'accord. Ça ne correspondait pas à ma vision. Au début, c'était pratique. } \\
\text { Ca me permettait de faire passer n'importe quoi. Ce n'est pas intéressant. } \\
\text { L'intérêt, c'est de faire venir d'autres gens. lly a un moment où ça a commencé } \\
\text { à ne plus discuter.» }\end{array}$ \\
\hline 3.7 & $\begin{array}{l}\text { Cognition, légitimité } \\
\text { et optimisation } \\
\text { consensus } \\
\text { Lâcher prise au nom } \\
\text { du consensus }\end{array}$ & $\begin{array}{l}\text { Président } \\
\text { + de } 10 \text { ans d'ancienneté } \\
\text { + de } 10 \text { salariés }\end{array}$ & $\begin{array}{l}\text { "Je suis à un stade, depuis } 3 \text { ou } 4 \text { ans, où je ne suis plus intéressé par le } \\
\text { résultat. Je regarde les processus. Ce qui m'intéresse, c'est la dynamique. Je } \\
\text { fais attention à ce que tout le monde s'exprime. Un tel fait ses prises de position } \\
\text { et je le défends.» }\end{array}$ \\
\hline
\end{tabular}

Note : les extraits d’entretiens ci-dessus évoquent les relations entre le président et l'assemblée générale à travers les différents moments dans la vie de l'association : dans le cadre de réunions en AG ordinaire ou extraordinaire, en conseil d'administration (émanation restreinte de l'AG) mais aussi lors de confrontations plus informelles. 
l'impossibilité d'optimiser simultanément différents principes d'efficience et de légitimité au profit de solutions forcément imparfaites à l'épreuve de l'action (Boncler \& Valéau, 2010). Nous formalisons à présent ces apprentissages psycho-cognitifs et sociocognitifs de la gouvernance et les positionnements retenus à travers la figure 1 et quatre propositions.

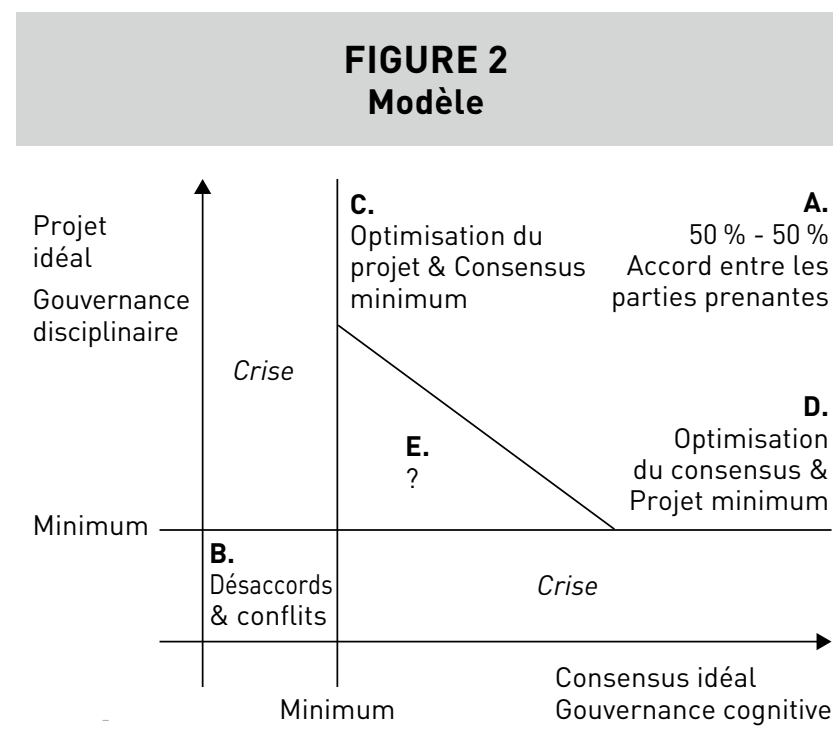

Proposition 1 : Les désaccords confirment l'impossibilité de maintenir, de façon permanente, une action collective unie autour d'un même projet (figure 2.A - 2.B).

Lorsque les présidents et les autres parties prenantes sont en accord, la gouvernance peut maintenir ce double idéal d'une action collective unie autour d'un même projet (figure 2.A). Tous les acteurs rencontrés ont ainsi évoqué une période idyllique de ce type. Cependant, tous considéraient également cette période comme révolue. Lorsque, tôt ou tard, un désaccord apparaît (figure 2.B), le président et son équipe se retrouvent dans une situation de crise. Le positionnement de ce moment, dans la figure 1, sous les deux minimums du projet et du consensus, signifie davantage le doute, la confusion et l'indétermination concernant la conduite à tenir qu'une absence totale de consensus ou de respect du projet porté par le président. Le président doit alors choisir entre une approche représentative de la gouvernance démocratique privilégiant la réalisation efficiente de l'idéal du projet (figure 2.C) et l'approche partenariale de la démocratie donnant priorité au consensus (figure 2.D). La zone E. de la figure 2 marquée d'un point d'interrogation pose la question de la possibilité d'établir une position intermédiaire face aux différends, celle-ci n'ayant fait l'objet d'aucune occurrence dans le cadre de notre échantillon. Nous retrouvons ce faisant un dilemme propre aux OBNL (Valéau, 2003). Le premier apprentissage lié à la dimension cognitive de la gouvernance consiste, pour le président, notamment, à accepter la difficulté, voire l'impossibilité d'accorder d'optimiser simultanément la réalisation efficiente de l'idéal du projet et le consensus idéal dans le cadre duquel le projet est légitimé par l'ensemble des parties-prenantes. En matière de gouvernance, ces désaccords confirment l'impossibilité de maintenir, de façon permanente, la fonction disciplinaire et la fonction cognitive des mécanismes intentionnels internes.
Proposition 2 : Certains présidents privilégient l'idéal du projet sous contrainte d'un minimum de consensus (figure 2.C)

Dans le premier scénario (figure 2.C), le plus fréquemment rencontré dans le cadre de nos entretiens, le président et son équipe se définissent eux-mêmes et se présentent comme les gardiens du projet de l'OBNL. Cette voie s'apparente à la gouvernance démocratique «représentative» (Blondiaux, 2008). En vertu de leur élection, les présidents et leurs équipes posent leur vision du projet comme le cadre de référence à l'intérieur duquel les autres acteurs et parties prenantes peuvent concevoir leurs participations. Ce faisant, toute question concernant les fondements du projet est perçue comme une menace pour l'OBNL. L'argument généralement mis en avant porte sur le caractère "anticonstitutionnel» des critiques émises. Partant de là, le président et son équipe identifient un danger justifiant des moyens d'exception, en particulier des formes d'exclusions provisoires ou définitives. L'apprentissage consiste ici à accepter des conflits plus frontaux associés à des options, à certains égards, plus ambiguës. L'exercice de la gouvernance partenariale est alors réduit à son minimum, il dépend alors de la compatibilité des points de vue exprimés avec cet idéal et devient ce faisant conditionnel. Cette voie questionne les limites que posent certaines OBNL à la liberté d'expression et de façon plus générale à la démocratie, réduite à son minimum. En matière de gouvernance, cette voie privilégie la fonction disciplinaire des mécanismes intentionnels internes, au détriment de leur fonction cognitive.

Proposition 3 : Certains présidents privilégient l'idéal consensuel. Ils garantissent le processus démocratique en renonçant à contrôler totalement le projet (figure 2.D).

Dans le second scénario (figure 2.D), relativement moins fréquent dans le cadre de notre échantillon, le président et son équipe veillent au maintien du consensus entre les parties prenantes. Cette voie se rapproche de la gouvernance partenariale décrite par Charreaux (2003) et, dans le contexte des OBNL, par Hoarau et Laville (2008) : les idées sont débattues plus ou moins longtemps avec un ensemble élargi d'acteurs et de parties prenantes, avant qu'une solution acceptable pour tous puisse être trouvée. L'apprentissage réside ici dans les règles régissant le processus, mais également dans les concessions que le président et son équipe ont à faire pour permettre cette forme plus poussée de démocratie. Dans ce scénario, le résultat obtenu importe moins que la façon dont il est produit. Ce choix consensuel a pour objectif d'éviter les conflits et les différends, en situant l'action partagée et la fraternité au-dessus de toute autre considération. Cette voie questionne cependant la capacité de certaines OBNL à porter un projet cohérent dans la durée, les grandes orientations de l'OBNL pouvant ainsi être critiquées et évoluer au gré des débats. En pratique, les limites au-delà desquelles le projet se retrouverait dénaturé ne sont plus définies par le président, mais socialement construites par une agora de parties-prenantes. En matière de gouvernance, cette voie privilégie la fonction cognitive des mécanismes intentionnels internes, en reléguant au second plan leur fonction disciplinaire.

Les arbitrages en faveur du consensus idéal semblent correspondre à une forme de lâcher prise de nature différente des arbitrages en faveur de l'idéal du projet. Ces derniers marquent une conviction dans le cadre d'un conflit cognitif concernant la 
vision, mais ils peuvent aussi être interprétés comme une forme d'intérêt personnel suivant lequel le président utilise ses marges de manœuvre pour privilégier sa propre vision du projet au détriment de celle des autres. Les deux présidents s'exprimant dans les deux derniers extraits du tableau 3 apparaissent plus ouverts à la discussion suivant une forme de maturité à travers laquelle ils ne semblent plus considérer que leurs définitions du projet seraient plus pertinentes que celles portées par les autres parties-prenantes. Ces deux présidents admettent avoir développé cette position à la suite de leurs précédentes expériences. Nous ajoutons ce faisant que le rôle cognitif joué par la diversité des parties prenantes (Charreaux, 2003; Herman \& Renz, 1997; Hoarau \& Laville, 2008) dépendrait en partie de l'ouverture d'esprit du président face à ces apports.

Proposition 4. Les formes prises par la gouvernance démocratique des $O B N L$ sont le résultat d'un arbitrage

Le principal apprentissage que nous formalisons sur la base de nos données empiriques consiste, pour le président, à mieux comprendre la nature du dilemme que font naître les désaccords et la nécessité d'arbitrer entre deux modes de production de la stratégie. Nous considérons tout d'abord un apprentissage psycho-cognitif au sens de Piaget (1971) et Watzlawick et al (1975) dans le cadre duquel le président passe d'un cadre de références fondé sur l'optimisation simultanée des critères d'efficience et de légitimité à une prise de conscience de l'impossibilité de cette double optimisation et de la nécessité d'arbitrer. Nous identifions là un changement des schèmes mentaux qui soustendent la logique de son raisonnement.

Cet arbitrage se prolonge ensuite sous la forme d'un apprentissage socio-cognitif concernant l'ensemble de la structure. Quelle que soit la voie retenue, le premier arbitrage du nouveau président et de son équipe constitue une jurisprudence initiale établissant auprès de l'ensemble des parties prenantes les modes opératoires destinés à réguler les désaccords durant son mandat. C'est à l'occasion de cette épreuve, qu'ils définissent leurs positions et établissent concrètement jusqu'où ils sont prêts à aller pour défendre le projet ou pour respecter l'exercice démocratique. Certains extraits d'entretiens présentés dans les tableaux 2 et 3 évoquent un retour à la normale, des habitudes et des façons de faire propres à l'OBNL. Ainsi, l'apprentissage, de nature plus collectif, réside également dans la rétention, la légitimation et l'institutionnalisation de ce premier arbitrage. La dimension cognitive de la gouvernance ainsi abordée détermine la façon dont le modèle partenarial (Charreaux, 2003; Haorau \& Laville, 2008) sera mis en œuvre. Une grande partie des cas de processus conflictuels établis par la littérature (Kelley et al., 2005; Hoarau \& Laville, 2008; Boncler \& Valéau, 2010; Kreutzer \& Jager, 2011) peuvent être interprétés en ces termes, avec presque toujours, après des périodes de conflits parfois très âpres, un retour à la normale. Le Tian et Venard (2012) proposaient une approche de la gouvernance dont les mécanismes seraient adaptés au type d'organisation, et notamment à sa culture. El Abboubi (2013) soulignait le caractère socialement construit du statut de partie prenante au sein de la gouvernance. Nous ajoutons quelques considérations supplémentaires propres aux OBNL : la diversité des formes prises par la gouvernance démocratique des OBNL est le produit de nombreux facteurs. Parmi ceux-ci, à travers cet article, nous mettons en avant l'importance des arbitrages effectués par les présidents. Nous ajoutons à la diversité des regards portés par les parties-prenantes sur le projet (Herman \& Renz, 1997; Valéau, 2003), la diversité des arbitrages mis en œuvre par les présidents pour établir leurs approches de la gouvernance démocratique.

Les arbitrages des présidents et de leurs équipes, illustrés dans le cadre de cet article, relient selon nous la gouvernance des OBNL aux sciences de gestion. Pour Laville (2010), l'associationnisme, c'est-à-dire cet espoir d'action collective citoyenne autour d'une cause commune, s'inspire des utopies, mais s'en distingue cependant par sa confrontation à la réalité de l'action. Nous abordons les sciences de gestion comme des sciences de la décision (Simon, 1947), étudiant comment les acteurs en situation de pouvoir mettent leurs valeurs à l'épreuve de l'action. Les sciences de gestion appliquées aux OBNL, ne consistent pas, comme on le présente trop souvent, à défendre une approche similaire aux entreprises. L'une des théories fondatrices des sciences de gestion reste la rationalité limitée : limitée par l'information, mais aussi comme on l'oublie parfois, par les préférences et les systèmes de valeurs des décideurs (Simon, 1947). Dans le cas présent, les présidents se retrouvent face à deux normes : l'idéal du projet et l'idéal consensuel. Leurs arbitrages constituent des apprentissages de la gouvernance (Hoarau \& Laville, 2008), mais aussi des innovations sociales (Richez-Battesti, Petrella \& Vallade, 2012) : ils permettent des renouvellements des formes de la gouvernance démocratique à travers de nouveaux modèles opérationnels d'action collective plus divers. Cette diversité constitue encore, malgré les processus de normalisation, selon Eynaud (2015) et Valéau (2003 et 2015), l'une des caractéristiques du secteur non lucratif.

L'une des limites de cette réflexion réside cependant dans notre focalisation quasi-exclusive sur les présidents, que ce soit à travers leur propre discours ou d'autres acteurs gravitant autour d'eux. Les arbitrages des présidents mis en exergue dans le cadre de cet article ne sont pas le seul facteur déterminant les formes prises par la gouvernance démocratique des OBNL. Même si ceux-ci constituent officiellement le sommet de la hiérarchie organisationnelle (Mintzberg, 1983), d'un point de vue sociologique, ces acteurs élus ne sont jamais que des parties prenantes parmi d'autres. Par ailleurs, le jeu de l'élection démocratique rend leurs prérogatives précaires, les majorités se faisant et se défaisant. Ainsi, nous retenons simplement la façon dont le président et son équipe organisent la gouvernance durant les périodes où ils restent en capacité de décider.

Loin d'épuiser le sujet de la gouvernance dans les OBNL, cet article se veut un jalon dans une analyse visant à comprendre les spécificités et les enjeux de ces organisations de façon à éviter un simple mimétisme des modes de gouvernance issus des entreprises. D'autres approches sont possibles, comme par exemple celle de Phanuel (2016) qui met le directeur au centre de l'OBNL. En l'absence de propriété, la question de qui se trouve légitimement au centre et en position d'arbitrage reste en partie indéterminée (Boncler \& Valéau, 2010). Certains considèrent qu'une partie importante de la gouvernance des OBNL se joue également dans la relation entre le président et le directeur. Suivant cette autre perspective, il conviendra d'intégrer davantage les problématiques liées aux besoins de professionnalisation des acteurs. 
Les futures recherches pourraient également prendre davantage en compte le poids des contextes. Bien que notre échantillon soit de taille trop modeste pour valider de quelconques corrélations, nous pouvons évoquer quelques pistes issues de nos données concernant les effets de la taille de l'OBNL, l'expérience du président, le secteur d'activité. Il semble que les préoccupations d'efficience deviennent plus prégnantes dans les grandes structures, cependant les arbitrages des présidents semblent également liés, comme précédemment évoqué à son expérience ainsi qu'au secteur d'activité, le secteur de l'éducation populaire apparaissant plus participatif. Cette relativisation de l'effet de la taille rejoint les constats effectués par Valéau (2015), dans le cadre de sa méta-analyse qualitative, selon lesquels les crises d'identité et le questionnement des priorités entre efficience et légitimité peuvent être posés à tous les stades du développement des OBNL et réorienter leur développement.

Enfin, il convient de rappeler que les OBNL ici étudiées étaient toutes françaises. D’autres recherches intégrant des OBNL d'autres pays permettraient d'identifier si les lois et les cultures nationales jouent un rôle dans les formes prises par la gouvernance démocratique des OBNL. Valéau (2015) montrait sur la base d'une méta-analyse comprenant des études de cas issus de plus de quinze pays différents que la plupart des OBNL passent par le même processus de développement mettant en tension des questions d'efficience et de légitimité. Nous faisons ici l'hypothèse que le dilemme posé dans le cadre de cet article entre gouvernance démocratique représentative visant l'idéal du projet et gouvernance démocratique participative visant un consensus idéal, se pose à la plupart des OBNL, quel que soit le contexte national considéré.

\section{Conclusion}

Selon Pareto (1906), la prise en compte de plusieurs critères fait que différentes combinaisons peuvent apparaître objectivement équivalentes, ce faisant seules les préférences humaines peuvent permettre de les départager. Cet article montrait les dilemmes dont les présidents font l'expérience, face aux désaccords, entre l'optimisation du projet sous contrainte d'obtenir une majorité et l'optimisation du consensus sous contrainte de respecter un minimum le projet fondateur. Nos analyses expliquent ainsi les malaises souvent ressentis par les présidents attachés à ces deux fondements des OBNL. Les résultats de notre étude montrent la difficulté, voire dans certains cas l'impossibilité de respecter simultanément et "également» l'idéal du projet et l'idéal consensuel. Nous espérons à l'issue de ce travail avoir donné aux lecteurs impliqués dans la vie des OBNL une perspective inspirée du terrain faisant écho à leur propre expérience.

En nous référant à la définition de Hoarau et Laville (2008) définissant la gouvernance des OBNL comme l'ensemble des mécanismes permettant la mise en cohérence du fonctionnement de l'organisation avec le projet, nous avons montré que cette mise en cohérence peut être obtenue de deux manières différentes : soit, suivant une approche disciplinaire de la gouvernance, en adaptant le fonctionnement sans dévier du projet défendu; soit, suivant une approche cognitive, en adaptant le projet de façon à maintenir un fonctionnement purement consensuel. A l'instar d'Eynaud (2015), après avoir donné aux acteurs des informations utiles pour éclairer leurs choix en matière de gouvernance, nous les renvoyons à leurs propres responsabilités : il leur appartient de décider rationnellement (Simon, 1983), quelle est la forme de gouvernance la plus adéquate, entre démocratie représentative et démocratie participative. La portée pratique de cet article s'arrête, délibérément, à une non-réponse retournant aux acteurs eux-mêmes la question du choix entre l'idéal du projet et le consensus idéal, entre démocratie représentative et démocratie participative à l'épreuve des désaccords. Il leur appartient de «reconstruire» cette cohérence évoquée par Hoarau et Laville (2008).

Notre étude mettait ainsi en avant une fonction de la présidence des OBNL que nous pourrions qualifier de «méta-gouvernance», consistant à assurer l'opérationnalité des mécanismes intentionnels internes, nécessitant, le cas échéant, de trancher entre leur fonction cognitive et leur fonction disciplinaire, suivant deux interprétations différentes de la démocratie associative. Nous relevons certaines ambiguités dans ce rôle du président l'amenant à gérer la démocratie dont il est lui-même issu. Il apparaît ainsi à la fois juge et partie, se retrouvant en position de réguler une gouvernance qui est elle-même supposée l'encadrer. Ce paradoxe constitue un aspect peu examiné du fonctionnement démocratique des OBNL.

\section{Bibliographie}

Biondi Y., Chatelain-Ponroy S., Eynaud S. \& Sponem S. (2010), "Quel modèle de gouvernance pour les associations?», Politique et Management Public, 27(1), p. 3-7.

Blondiaux L. (2008), Le nouvel esprit de la démocratie. Actualité de la démocratie participative. Paris, Le Seuil.

BonCler J. \& VAlÉAu P. (2010), Créer et manager une association loi 1901, Éditions De Boeck.

Bouglet J. \& Joffre O. (2015), «Les priorités du dirigeant face à ses parties prenantes : l'apport d'une approche centrée sur les attentes ", Management international, HEC Montréal, 20 (1).

Callon M., Lascoumes P. \& Barthe Y. (2001), Agir dans un monde incertain. Essai sur la démocratie technique. Paris, Le Seuil.

Charreaux G. (1997), Le gouvernement des entreprises, Paris, Economica.

Charreaux G. (2003), «Quelle théorie pour la gouvernance? De la gouvernance actionnariale à la gouvernance cognitive», in Allouche J., Encyclopédie des Ressources Humaines, Paris, Vuibert.

Charreaux G. (2006), «Les théories de la gouvernance : de la gouvernance des entreprises à la gouvernance des systèmes nationaux", in Charreaux G. et Wirtz P., Gouvernance des entreprises Nouvelles perspectives, Paris, Economica.

Defourny J. \& Nyssens M. (2010), Approches européennes et américaines de l'entreprise sociale : une perspective comparative, Colloque 2010, ADDES (Association pour le développement de la documentation sur l'économie sociale).

Denzin N.K. \& Lincoln Y.S. (1994), Handbook of Qualitative Research, Sage.

DruCKer P. (1990), Managing the Nonprofit Organization, Harper, New-York.

Eynaud P. (2015), Pratiques de gouvernance : entre diversité et normalisation, Collection Hors-Série Juris Association, Editeur Dalloz. 
El Аввочвi M. (2013), «Comment identifier les parties prenantes dans une certification RSE?", Management International, Vol. 17, N², p. 48-62.

Eynaud P., Chatelain-Ponroy S. \& Sponem S. (2015), Panorama et typologie des modes de gouvernance des associations, in Philippe Eynaud. La gouvernance entre diversité et normalisation, Dalloz, jurisEditions, p. 22-42.

Fontana A.\& Frey J. (1994). «Interviewing, the Art of Science» in The Handbook of Qualitative Research, N. a. Y. L. Denzin. Thousand Oaks : Sage

Freeman R.E. (1984), Strategic Management: A Stakeholder Approach, Pitman, Boston.

Glaser B. \& STrauss A. (1967), The discovery of grounded theory, strategies of qualitative research, Aldine Publishing Company.

Habermas, J. (1989). The structural transformation of the public sphere. MIT: Cambridge

Herman R. D. \& Renz, D.O. (1997), « Multiple constituencies and the social construction of non-profit organization effectiveness", Nonprofit and Voluntary Sector Quarterly, 26, 185-206.

Herman R.D. \& Renz, D.O. (2008), «Advancing nonprofit organizational effectiveness research and theory: Nine theses", Nonprofit Management \& Leadership, 18(4), p.399-415.

Hoarau C. \& LAVILle J.-L. (2008), La Gouvernance des associations, Sociologie économique, Toulouse, ERES.

Kelley M., Lune H. \& Murphy S. (2005), "Doing syringe exchange», Nonprofit and Voluntary Sector Quarterly, 34(3), p. 362-386.

Koeble M. (2006), Le pouvoir local ou la démocratie improbable, Paris, Editions de Croquant.

Kreutzer K. \& Jager U. (2011), «Volunteering vs managerialism», Nonprofit and Voluntary Sector Quarterly, N40, p. 634-661.

Laville J.-L. \& Sainsaulieu R. (1997), Sociologie de l'association, Desclée, Paris.

LAVILLE J.-L. \& GLÉMAIN P. (2009), L’ESS aux prises avec la gestion, Desclée, Paris.

Laville J.-L. (2010), Politique de l'association, Seuil, Paris.

LaVille J.-L. \& SAinsaulieu R. (2013), L'association, Sociologie et économie, Pluriel.

Laville J.-L., Young D. \& Eynaud P. (2015), Civil society, The Third Sector, Social Enterprise: Governance and Democracy, Routledge Publisher Oxfordshire, Royaume Uni.

Laville J.-L. (2016), L'Économie sociale et solidaire, Pratiques, théories, débats, Seuil, Paris.

Lecy J. D., Schmitz H. P., \& Swedlund H. (2012), «Nongovernmental and not-for-profit organizational effectiveness: A modern synthesis", Voluntas: International Journal of Voluntary and Nonprofit Organizations, 23(2), p. 434-457.

Le Tian C. \& Venard B. (2012), «Pour une approche contingente de la gouvernance», Management International, 16 (2), p. 25-38.
Mayaux F. (1996), Noyau stratégique des associations, Thèse de Doctorat, Université Lyon 3.

Meier O. \& Schier G. (2008), «Quelles théories et principes d'actions en matière de gouvernance des associations?", Revue management et avenir, 2008/6, $\mathrm{N}^{\circ} 20$, p. 179-198.

Mitchell R.K., Agle B.R. et Wood D.J. (1997), «Toward a Theory of Stakeholder Identification and Salience: Defining the Principle of Who and What Really Counts", Academy of Management Review, $22: 4$, p. 853-886.

Mintzberg H. (1983), Power in and around organizations, Prentice-Hall.

Organisation des Nations Unies, Hopkins (2003),

Pareto V. (1906), Manuale d'economia politica, Milano : Società editrice libraria, $1^{\text {ère }}$ édition française, Paris : Giard et Brière 1909, réédition in Euvres complètes de Vilfredo Pareto, Genève : Droz, 1966.

Phanuel D. (2016), « Le management relationnel d'une association de l'économie sociale et solidaire», Management International, Vol. 20, N³, p. 105-121.

Reid W. \& KARAmbayya R. (2009), «Impact of dual executive leadership ", Human Relations, $\mathrm{N}^{\circ}$ 7, p. 1073-1112.

Richez-Battesti N., Petrella F. \& Vallade D. (2012), «L'innovation sociale une notion aux usages pluriels : quels enjeux et défis pour l'analyse», Innovations, p. 15-36, Nº35.

Rothschild J. \& Stephenson M. (2009), «The Meaning of Democracy in Non-profit and Community Organizations Charting the Currents of Change ", American Behavioral Scientist, Vol. 52, Nº, p. 800-806.

Rousseau F. (2007), «Réapprendre à conter : genèse d'un entrepreneur social», Gérer et comprendre, N 87, p. 23-34.

SAlAmon L.M. \& ANHEIER H.K. (1992), «In search of the non-profit sector. I: The question of definitions», Voluntas, N³, p. 125-151.

Simon H. (1947), Administrative Behavior: a Study of DecisionMaking Processes in Administrative Organization, MacMillan.

Simon H. (1983), Administration et processus de décision, Economica.

Toé M. (2014), «Les déterminants individuels du plafond de verre : cas de la composition des instances de gouvernance des entreprises françaises cotées», Management International, Vol. 18, N 4, p. 48-67.

VALÉAu P. (2003), «Différentes manières de gérer les associations », Revue Française de Gestion, N 146, p. 9-21.

Valéau P. \& Annette F. (2012), La gestion des «déviances» des associations par les pouvoirs publics : une autonomie conditionnelle», Management International, 16(3), p. 139-152.

VAlÉAu P., PARAK H. \& Louart P. (2013), «Les équipes associatives entre rationalité en valeur et entrepreneuriat social », RECMA, $\mathrm{N}^{\circ} 330$, p. 89-106.

Valéau P. (2015), "Stages and Pathways of Development of Nonprofit Organizations: An Integrative Model», Voluntas, Vol. 26, Issue 5, p. 1894-1919. 